\title{
Un testo di "micronarrazioni" e alcune note in forma di quesiti
}

\author{
Carla De Bellis
}

La micro-narrazione, ovvero Nuovi progetti di narratori italiani è il titolo dell'antologia che Carlo Marcello Conti e Lamberto Pignotti hanno curato per la rivista Zeta (edita ad Udine da Campanotto), facendone il complemento dell'antologia apparsa due anni prima col titolo Il "nuovo" in poesia in un numero monografico della stessa rivista, dove, seguendo il criterio dell'inchiesta, Conti e Pignotti hanno interrogato circa un centinaio di poeti chiedendo loro alcuni testi poetici e una breve nota di commento o di riflessione sulla loro stessa scrittura.

I narratori presenti in questa antologia sono poco più di quaranta, $\mathrm{i}$ loro saggi sono brevi racconti, frammenti isolati o brani di romanzi, e in quasi tutti i casi una Nota dell'autore vi aggiunge degli elementi di riflessione.

Una simile abbondanza di materiale che, essendo molto disomogeneo, apre a ogni passo i varchi e le lacune della discontinuità, non può non stimolare un desiderio come di far ordine, di tentarne una descrizione e, comunque, di intervenire o piuttosto di reagire dialogicamente.

La forma dell'intervento critico in questo caso non può essere, allora, che interrogativa.

C'è, intanto, da andare alla radice del problema e da chiedersi se un discorso critico o anche una descrizione ragionata del materiale dell'antologia siano legittimi e possibili, dato che critica e descrizione sono operazioni che, per quanto dinamiche, tendono a fissare l'oggetto esaminato e a bloccarlo in una definizione. Mentre la volontà di esperimento e di ricerca di queste scritture, per di più molto varie, le rende ribelli a qualunque forma di costrizione, e la loro ricchezza è nella tensione anarchica e nel desiderio, che le accomuna, di proiettarsi nel vuoto della novità e nell'incertezza dell'esperibile.

Ogni discrimine critico potrebbe essere quella "spiegazione micidiale" temuta da Celati, il laccio che trattiene la fuga avventurosa della scrittura e ne soffoca la fertile aleatorietà.

Ma, d'altro canto, se questa è la scrittura dell'"abbandono," del "rischio," del "gioco," è anche quella che nel "deserto" della storia e nello spazio dilatato dell'esterno e dell'alieno traccia tuttavia dei "percorsi obbligati" (come ancora Celati indica nella "conversazione" riportata all'inizio dell'antologia) e cerca la linea della parabola per fermare, secondo la necessità della consapevolezza e della riflessione, la dispersione dell'esperienza. 
Se dietro tutto questo c'è ludus, sarà allora quello della lotta, dell'agone, che entro uno spazio simile a un arena si disputa tra la scrittura e la comunque inscindibile riflessione. Ed è nel punto mediano, in quello dell'incontro, che il discorso critico può insinuarsi, aggiungendo sfaccettature alle posizioni della scrittura e del suo riflesso. Si parla della natura intrinsecamente dialogica della prosa narrativa in alcune pagine di Bachtin (83-93), cosi come si parla di "raggi," di "sfaccettature" e "angoli diversi" che l'immagine dell'oggetto del discorso presenta, e inoltre della "molteplicità di cammini. strade e sentieri" comc pure dell'“arena" di un incontro, non sommesso né pacifico quindi: il duplice incontro della parola narrativa con tutte le parole altrui che hanno già nominato il suo oggetto e quello con tutte le parole che le opporrà la risposta dell'ascoltatore.

Ecco allora che l'enunciazione critica può non essere soltanto la voce esterna, fuori campo, astratta dalla lotta vitale della parola, ma può forse inserirsi in quel "medium elastico" che la parola attraversa, può essere un ulteriore stimolo interlocutorio, una delle sue attese o una delle posizioni (le figure) della sua lotta.

La parola, dunque, è espressa nella dinamica di un dialogo e nella divisione di un dilemma dalle molteplici facce (il riflesso, l'oggetto, la parola aliena, l'ascolto interlocutorio), e inoltre da un percorso plurimo e radiante è tentata fuori di sé.

Infatti, fin dal titolo del suo scritto, Pignotti avverte che sono in gioco "tutte le direzioni," e poi accumula gli interrogativi insoluti di una scrittura di ricerca e allinea gli spazi artificiali, scenici, che la riflessione via via le apre davanti per controllarne il percorso.

Ma prima di tentar di accedere in quel varco che discrimina la duplicità della scrittura - di queste, particolari, forme di scrittura - , occorre considerare alcune peculiarità dell'antologia nel suo complesso e riferirsi a quanto Pignotti e Guzzi già indicano nelle pagine introduttive.

- Il fatto innanzitutto che si tratti di un'autoantologia. Il gesto di scrivere per l'antologia o di ritagliare un frammento della propria scrittura presuppone, da parte dell'autore, l'esercizio di un'ottica autoriflessiva e autovalutativa, sottolineata inoltre dalle Note a piè di pagina: il pezzo quindi, a prescindere dall'interno grado di consapevolezza, è comunque calato entro un alone critico e dilatato da una specie di sua aura riflessiva.

- La micronarrazione. Qualunque sia il motivo pratico della scelta di tale ridottissima dimensione, questa comporta in linea di massima l'idea della virtù del linguaggio e quella della irrilevanza della storia ("per non raccontare una storia," avverte Conti).

- La tensione progettuale degli scritti: che implica il loro deliberato comporsi in forma non definitiva, perfettibile e dinamica.

- E infine l'iconicità, a vari livelli, di molti testi, che, oltre a qualificare formalmente in vario modo il linguaggio, sembra rinviare quasi a un gusto artigianale del testo, che ne fa un prodotto da costruire e percepire secon- 
do una dimensione sensoriale plurima, foggiandolo con una più concreta fattura, attenta anche alla fisicità della pagina scritta e di quanto essa trasmette. Guardando più da vicino $\mathrm{i}$ testi, è inevitabile reperire le sparse tracce del ludus bellico della scrittura, il suo gioco di riflessi e la duplicità dialettica che la divide (come nel caso del Pugile di Annino).

Sarà il margine dello scontro, la superficie dello specchio o il segno della frattura, in ogni caso il tormento che non smette, ambiguamente, di sedurre la scrittura è la necessità e l'angoscia del limite. Tutto quanto, cioè, le dà veste identità forma, ma la allontana dal suo oggetto, aprendole nello stesso tempo il varco verso di esso. Duplice soglia tra la scrittura e l'esterno, il suo oggetto, e tra la scrittura e l'interno, la sua immagine riflessa.

Alcuni testi propongono direttamente una struttura dialogica, avvertendo della natura emblematica del dialogo attraverso la qualità surreale del linguaggio (Calzolari).

La scrittura è spesso al centro di un dilemma che vede in campo "forze antagoniste" e ossimori non conciliati. Nel difficile punto mediano il linguaggio oscilla, sempre tentato dal dubbio e dal silenzio (Dorigo, Tommasi).

Il limite, perseguito e insieme temuto, è ciò che discerne gli opposti, ma è anche lo schermo che divide il gesto dal fantasma della forma. La tentazione di infrangerlo apre varchi surreali e genera il fenomeno del personaggio del romanzo che si trasforma in essere reale (Battilana), o quello dell'autore che assume la natura della scrittura stessa integrando catene di opposti in un paesaggio dove spine, frecce e ferite alludono invece a una dolorosa lacerazione (Di Scalzo).

La qualità alterna e discriminante della scrittura emerge anche visivamente negli spazi bianchi, i silenzi e le lacune che la dividono, quasi cancellando la sintassi del racconto (Ermini).

S'allenta ugualmente il legame degli oggetti con la tecnica opposta della scrittura che dilaga lungo un flusso indifferenziato, perché ciò che in tal modo si nega è la distribuzione gerarchica della realtà, proposta invece in una sua continuità quasi materica, che la sottragga al discrimine e alla discontinua aggregazione (Docimo).

Non credo sia casuale la ricorrenza, in alcuni testi, della metafora del buio e della luce, del bianco e del nero, antagonisti e insieme stretti ossimoricamente, ad indicare la scrittura che solo a patto di scavare neri segni verga il biancore silenzioso del foglio, in un gesto di continua mediazione (Dorigo, Morandini).

Sul filo di una duplice parola, che cerca la sua sintassi nel legame fra tempi e spazi diversi, si può entrare e uscire dall'interno all'esterno, dal presente alla memoria (Fergnani, Grazioli, Riberto). La parola narrativa può assumere l'energia centripeta del linguaggio poetico e precipitare piani ed oggetti verso un centro che appare motore del traslato, dislocando altrove il suo senso (Serrao).

Oppure può dichiarare i suoi "due luoghi," mostrando lo spazio retorico nell'atto di intersecarsi con lo spazio dell'oggetto, e la profondità prospettica 
degli oggetti nell'atto di spostarsi sul piano della scrittura (Falasca).

Nominando metaretoricamente le sue forme, la scrittura arriva ad assumere quasi la veste di un personaggio antagonista o di un paesaggio alternativo (Pinni. Ferjancic). Sarà a voltc una precisa figura del linguaggio a riempire, in luogo dell'oggetto, lo spazio c a contraddire il racconto: ad escmpio, la sineddoche, forse a indicare la parzialità di un discorso che dispera di cogliere un frutto sensato e omogeneo (Gaudio, Di Marco). Così come l'esercizio di un lipogramma non è solo uno specchio parodico della parola, ma, giocando - in negativo - su un'assenza, ne richiama il volto oscuro e le tensioni frustrate (Weiss).

Dove la parodia, invece, si fa metanarrazione e non solo metalinguaggio, si fa cioè artificiale riflesso dell'artificio del racconto, la scrittura si mostra nell atto di inseguire se stessa lungo la curva di un percorso tautologico (Cavallo, Adolgiso).

La scrittura come generatrice di artifici formali si assimila all'occhio che appetisce l'immagine e coglie la dimensione dello spazio. In alcuni testi essa si fa occhio plasmarte e chiama alla luce plastici oggetti, immaginando uno spazio circolare dove centro e periferia continuamente si implicano e luce e buio si modellano a vicenda (U.B. Conte).

Oppure sulla pagina si allineano gesti e oggetti minuti che la parola uno dopo l'altro evoca e impone, come un occhio, appunto, che contemporaneamente vede e plasma (Di Iorio).

Un'ultima nota.

In più di un testo compare un termine: barocco (ad esempio nella Nota di Lunetta), e più di un testo esibisce una lussureggiante ridondanza verbale, un lessico sensuoso e un gusto per la dovizia degli aggettivi usati come un materiale plastico che foggia l'oggetto per aggiunte progressive (Lanuzza, Molli, Sproccati).

Da una simile scrittura l'oggetto emerge come da uno spazio immaginario con la sua natura di sensibile artificio.

C'è allora da chiedersi: tra gioco, parodia, metalinguaggio e barocco, la riflessione sulla scrittura, vedendola disperare del proprio oggetto, la sta forse orientando al gusto, non più solo provocatorio e polemico, della forma? E se l' "indagine" di cui parla Carla Vasio nel passo tratto dal suo romanzo adom. brandovi l'attitudine interrogativa della scrittura, è, verso l'oggetto, aleatoria, si ripiegherà la parola in una voluta barocca?

\section{Universilà di Roma "La Sapienza"}

\section{OPERE CITATE}

Bachlin, Michacil. Estetica e romanzo. Torino: Einaudi, 1979.

Conli, Carlo Marcello, e Lamberto Pignotti. "Il "nuovo" in poesia." Zela 9 (1986).

"Nuovi progelli di narratori italiani." 7e'la 10 (1988). 